\title{
Proportional Fair Scheduling in Hierarchical Modulation Aided Wireless Networks
}

\author{
Xuan Wang and Lin Cai, Senior Member, IEEE
}

\begin{abstract}
Theoretically, superposition coding (SPC) can achieve the capacity of a degraded Gaussian broadcast channel. A practical implementation of SPC, hierarchical modulation (HM), has recently been adopted in industry. Using HM, how to explore the multi-user diversity gain in a time-varying wireless environment to maximize throughput and maintain fairness is an open issue. Using greedy opportunistic scheduling algorithms will lead to a severe starvation problem. In this paper, we study the proportional fair scheduling (PFS) problem in an HMaided wireless network, jointly considering the user selection and utility maximization problems. Shannon capacity based and practical HM based optimal scheduling problems are formulated. An optimal algorithm and a low complexity suboptimal algorithm are proposed to solve the practical scheduling problem combining the opportunistic PFS and HM. Simulation results demonstrate that the proposed algorithms can achieve $50 \%$ to $100 \%$ throughput gain compared to the single-user opportunistic PFS solution depending on the number of users and have better fairness performance than the existing single-user and HM-based solutions.
\end{abstract}

Index Terms-Superposition coding, proportional fair scheduling, hierarchical modulation, multiuser scheduling.

\section{INTRODUCTION}

W IRELESS channels are time-varying and broadcast in nature. How to optimize wireless scheduling algorithms to maximize system efficiency and ensure fairness, considering the wireless channel characteristics, is both challenging and promising.

Traditionally, a scheduler (in the link layer) divides the wireless resources into orthogonal logic links. For each logic link, the physical (PHY) layer deals with channel impairments (e.g., fading, shadowing, path loss) aimed to maximize the spectrum efficiency under certain bit error rate (BER) constraint. Using the services provided by the PHY layer, upper layer protocols can be designed without considering the wireless channel characteristics.

Such layered solutions are not most efficient. In [1], an opportunistic scheduling was proposed for multiple users with independent, time-varying channel conditions sharing the uplink in a cellular network. The scheduler avoids to select the users in deep-fading to transmit to improve the system efficiency. Instead of concealing the fast-fading in the PHY layer, the opportunistic scheduling utilizes the randomness

Manuscript received February 22, 2012; revised August 19 and November 27, 2012; accepted January 10, 2013. The associate editor coordinating the review of this paper and approving it for publication was L. Sanguinetti.

The authors are with the Department of Electrical and Computer Engneering, University of Victoria, Victoria, British Columbia, Canada (e-mail: \{mythjill, cai\}@ece.uvic.ca).

Digital Object Identifier 10.1109/TWC.2013.022013.120266 of channel conditions to improve system performance. This approach has also been extended for the downlink case in [2].

To ensure fairness among competing users, the proportionally fair (PF) rate allocation for wired networks was proposed in [3]. Using the channel state information (CSI) from the receiver, the opportunistic PF scheduling (PFS) algorithm for wireless networks was proposed in [4].

On the other hand, for broadcast wireless channel, superposition coding (SPC) was proposed to transmit a symbol containing information bits for users with different signal to noise ratio (SNR) simultaneously, which can achieve the capacity bound of a degraded Gaussian broadcast channel [5]. Recently, SPC has been implemented as hierarchical modulation (HM) using embedded constellation, which requires CSI from the receiver to select the suitable modulation.

Ideally, a wireless scheduler should exploit the multi-user diversity and the spatial diversity gain (using HM) while maintain fairness (using opportunistic PFS). Since the opportunistic PFS and HM both need the CSI feedback in a similar frequency in a block fading channel (per-block feedback), using these two technologies together, system performance can be improved by taking the advantage of both gains without increasing signalling message complexity. However, how to design an efficient and fair scheduler for SPC or HM-aided wireless networks is an open, challenging issue. When using SPC, the power allocation and performance of one user will be affected by the other user sharing the channel simultaneously. Thus, user selection and resource allocation should be jointly optimized. It is even more challenging when considering the HM implementation, since the best solution using the theoretical Shannon capacity-based analysis may not be feasible in practice, which motivates this work.

The main contributions of this paper are three-fold. First, we formulate the two-user opportunistic PFS scheduling problems: an SPC-based theoretical problem using Shannon capacity and an HM-based practical problem. Second, we propose an optimal algorithm and a suboptimal algorithm to solve the practical scheduling problem using opportunistic PFS and HM. Third, extensive simulations have been conducted to evaluate the performance of the proposed algorithms. Simulation results have demonstrated that the proposed algorithms can achieve substantial throughput gain compared to the existing single-user PFS solution and have better fairness performance compared to the existing HM based solutions.

The rest of the paper is organized as follows. Sec. II introduces the background information and the related work on SPC, HM and PFS. In Sec. III, the system models are presented and the scheduling and utility maximization problems 
are formulated. The optimal and suboptimal scheduling algorithms are proposed in Sec. IV, followed by the performance evaluation in Sec. V. Concluding remarks and further research issues are discussed in Sec. VI.

\section{Preliminaries and Related Work}

\section{A. Proportional Fair Scheduling}

In a wireless network, as the channels of different users are independent and heterogeneous, opportunistic scheduling was proposed to exploit the multi-user diversity gain to improve system efficiency [4], [24]. In a practical wireless system, a greedy algorithm that selects the user with the best channel quality to transmit tends to always select the users near the base station (BS) which is unfair and leads to the starvation problem.

The PFS was proposed to make the tradeoff between the multi-user diversity gain and fairness. A scheduling policy $\mathcal{P}$ is proportionally fair, if and only if the sum of the logarithmic average user throughput is maximized after the scheduling decision [4]:

$$
\mathcal{P}=\underset{\mathcal{S}}{\arg \max } \sum_{i \in U} \log R_{i}^{(\mathcal{S})}
$$

where $U$ is the active user set and $R_{i}^{(\mathcal{S})}$ is the average throughput of user $i$ under scheduling policy $\mathcal{S}$.

If only one user is allowed to transmit in any time slot $t,(1)$ is degenerated to selecting the user with the largest $r_{i}(t) / R_{i}(t)$ among all active users, where $r_{i}(t)$ is the instantaneous rate of user $i$ in slot $t$, and $R_{i}(t)$ is its average throughput before slot $t$. Typically, the average throughput can be updated by an exponentially weighted moving average algorithm [4]:

$R_{i}(t+1)= \begin{cases}\left(1-\frac{1}{T}\right) R_{i}(t)+\frac{1}{T} r_{i}(t), & \text { for } i=i^{*}, \\ \left(1-\frac{1}{T}\right) R_{i}(t), & \text { for } i \neq i^{*},\end{cases}$

where $T$ corresponds to the window size to smooth the throughput, and $i^{*}$ is the index of the scheduled user at time $t$. To simplify the notation, we omit $(t)$ in $R(t)$ and $r(t)$ hereafter.

Similar to the results in [11] (which extended the PFS to a multi-carrier system), if in a system where multi-users can transmit simultaneously, the proportional fair scheduling policy $\mathcal{P}$ should satisfy

$$
\mathcal{P}=\arg \max _{\mathcal{S}} \prod_{i \in U_{S}}\left(1+\frac{r_{i}}{(T-1) R_{i}}\right),
$$

where $U_{S}$ is the set of the selected users by scheduling policy $\mathcal{S}$.

\section{B. Superposition Coding}

Superposing signals for multiple users to achieve the capacity of degraded Gaussian broadcast channel was first introduced in [5] and named superposition coding (SPC). SPC is of great interest to enhance the network performance in various scenarios [6]-[10]. By using SPC together with successive interference cancellation (SIC), the capacity bound of a downlink degraded Gaussian broadcast channel can be achieved [12]. In a general order $\left|h_{1}\right| \leq\left|h_{2}\right| \leq \ldots \leq\left|h_{N}\right|$, where $\left|h_{i}\right|$ is the channel gain of user $i$, the capacity bound of user $i$ is defined by

$$
r_{i}=\log _{2}\left(1+\frac{P_{i}\left|h_{i}\right|^{2}}{N_{0} B+\sum_{j=i+1}^{K} P_{j}\left|h_{i}\right|^{2}}\right) \mathrm{bps} / \mathrm{Hz},
$$

where $P_{i}$ is the power allocated to user $i, N_{0}$ is the noise spectral density and $B$ is the channel bandwidth.

\section{Related Work}

Although SPC and PFS have been proposed in 1970's and 1990's, respectively, there is very limited cross-domain work on the combination of these two powerful techniques. Recently, an implementation of SPC, called HM, has been adopted in Digital Video Broadcasting (DVB) and several other standards [13], which generates new interests in this promising area.

Different from the SPC-related problem [25], the scheduling problem for $\mathrm{HM}$ is more difficult due to the discrete feature of the number of bits allocated and their BER requirements. In [14], [15], two opportunistic scheduling algorithms were proposed for a wireless network using two-layer HM, which allows two users to transmit simultaneously, namely twobest-user opportunistic scheduling (TBS) and hybrid twouser opportunistic scheduling (HTS) respectively. TBS selects two users with the first and second highest channel gain to transmit; HTS selects the first user with the highest channel gain and the second user with the highest relative channel gain, defined as the instantaneous channel gain normalized to its short-term average channel gain. TBS can be viewed as a direct extension of the single-user throughput-maximized opportunistic scheduling, and HTS is aimed to achieve a better max-min fairness. However, the bit allocation scheme in TBS/HTS may not fully explore the benefit of SPC/HM, since the constellation size is determined by the first user only.

In [16], a hybrid two-user opportunistic scheduling algorithm named HTS2 was proposed. It selects the first user based on the proportional fairness and the second user based on whether there are higher channel gain users in the same subcarrier. After user selection, transmission power is reallocated among the selected users to achieve better fairness.

The above HM-based scheduling algorithms separated the user selection and power allocation, which cannot achieve proportional fairness. In [17], an analytical approach was given to study PFS with SPC, jointly considering user selection and power allocation according to the sum-rate gain. Since multi-user PFS is not necessarily lead to a sum-rate gain, the resultant user selection may deviate from the optimal one based on multi-user PFS.

\section{System Model AND Problem Formulation}

\section{A. System Model}

We consider a two-layer SPC-aided single-cell wireless cellular network. The channel is assumed to be a quasi-static flat fading channel, i.e., in each time slot, the channels are static and independent of each other, and among different time slots, the channel fading follows a specific probability distribution (such as Rayleigh fading). We focus on the downlink case, and assume that the BS can obtain the instantaneous CSI for each slot. 


\section{B. Proportional Fair Scheduling Problem}

With HM, a scheduler can allocate a slot to at most two receivers. According to (3), the PFS problem is to find the user pair $\left(i^{*}, j^{*}\right)$ that satisfies $\left(i^{*}, j^{*}\right)=\arg \max _{(i, j)} U_{(i, j)}$ at each time slot, where $U_{(i, j)}$ is the PF-utility of the selected user pair $(i, j)$.

Based on (3), we define $U_{(i, j)}$ as

$$
U_{(i, j)}= \begin{cases}\left(1+\frac{r_{(i, j)}^{i}}{(T-1) R_{i}}\right)\left(1+\frac{r_{(i, j)}^{j}}{(T-1) R_{j}}\right), & \text { if } i \neq j, \\ 1+\frac{r_{(i, j)}^{i}}{(T-1) R_{i}}, & \text { if } i=j,\end{cases}
$$

where $r_{(i, j)}^{i}$ is the instantaneous rate of user $i$ when user pair $(i, j)$ is selected. For $i \neq j$, w.l.o.g, user $i$ is assumed to have a higher or equal channel gain than user $j$.

\section{Theoretical Capacity Based PF-Utility Maximization Prob- lem}

First, we consider the theoretical Shannon capacity based PF-utility maximization problem when using PFS in SPCaided wireless networks.

The PF-utility maximization problem is to maximize the PF-utility function (5) where the instantaneous rates lie in the capacity region. Since the capacity region is a closed convex set and the PF-utility function (5) is also convex, the problem is to maximize a convex function on a closed convex set. The maximum can be obtained in the boundary of the convex set [18]. Thus, for our problem, full power should be allocated to maximize the PF-utility.

Define the channel SNR of user $i$ as $\gamma_{i}=P\left|h_{i}\right|^{2} / N_{0} B$, where $P$ is the system power constraint. Based on (4), the instantaneous rates of paired user $i$ and $j$ can be written as, respectively,

$$
\begin{aligned}
& r_{(i, j)}^{i}=\log \left(1+q_{(i, j)}^{i} \gamma_{i}\right), \\
& r_{(i, j)}^{j}=\log \left(1+\gamma_{j}\right)-\log \left(1+q_{(i, j)}^{i} \gamma_{j}\right),
\end{aligned}
$$

where $q_{(i, j)}^{i}=P_{i} / P$ is the portion of power allocated to user $i$.

For $i \neq j$, by substituting (6) and (7) into (5), after some manipulations and simplifications, the PF-utility maximization problem is formulated as follows.

Problem 1:

$$
\begin{aligned}
\max & \frac{\log \left(1+q_{(i, j)}^{i} \gamma_{i}\right)}{R_{i}}-\frac{\log \left(1+q_{(i, j)}^{i} \gamma_{j}\right)}{R_{j}} \\
& +\frac{\log \left(1+q_{(i, j)}^{i} \gamma_{i}\right) \log \left(\frac{1+\gamma_{j}}{1+q_{(i, j)}^{i} \gamma_{j}}\right)}{(T-1) R_{i} R_{j}}, \\
\text { s.t. } & 0 \leq q_{(i, j)}^{i} \leq 1 .
\end{aligned}
$$

When $q_{(i, j)}^{i}=0$ or 1 the Problem 1 also includes the case that a single user is scheduled.

Due to the duality of Gaussian multiple-access and broadcast channels [19], Problem 1 also formulates the two-user SIC based multi-user uplink scheduling problem when the sum uplink power is fixed. In practice, individual power constraint is a more realistic assumption, and our approach is not directly applicable and needs further extension.

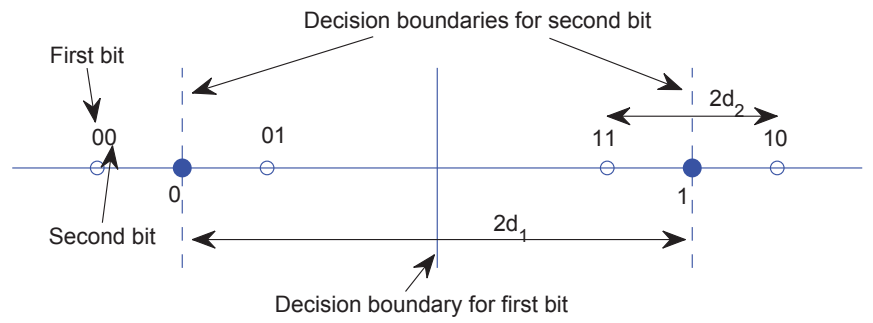

Fig. 1. 2/4-HMPAM with Gray mapping. The filled circles represent the fictitious symbols which are not actually transmitted. The open circles represent the real transmitted symbols. The digits attached to the symbols represent the bits information of the symbols (real or fictitious).

\section{HM-Based PF-Utility Maximization Problem}

The optimal solution of Problem 1 may not be feasible in a practical system, and an uncoded HM based system is considered in this subsection. Since in an uncoded system with block fading, BER and BLER (Block Error Rate) have a direct one-to-one mapping, in the following, we only consider the PF-Utility maximization in the symbol level.

The HM-based PAM (or QAM) is a generalized PAM (or QAM) with flexible Euclidean distance among constellation points. Here, we consider a system deploying two-layer HM based square QAM (HMsQAM) with Gray mapping, which has been well investigated in [20] and the reference therein. Since square QAM can be viewed as two identical and orthogonal PAM modulations, we first analyze the two-layer HM based PAM (HMPAM) with Gray mapping, having $n$ bits in the first layer and $m-n$ bits in the second layer, named $2^{n} / 2^{m}$-HMPAM.

As discussed in [20], a $2^{n} / 2^{m}$-HMPAM has $m$ levels of constellation points. The constellation points in level- $i(i<m)$ are fictitious and represent the symbols corresponding to the $i$-th bit. The constellation points in level- $m$ represent real symbols. The Euclidean distance between the constellation points in level- $i$ is $2 d_{i}$. The first $n$ bits belongs to the first layer and the rest $m-n$ bits belong to the second layer. Within each layer, we have $d_{i}=2 d_{i+1}$ (here level- $i$ and level- $(i+1)$ belong to the same layer.). A sample of 2/4-HMPAM is shown in Fig. 1, which has two layers and also two levels.

The Euclidean distance between the constellation points in level- $i$ of $2^{n} / 2^{m}$-HMPAM is

$$
2 d_{i}= \begin{cases}\left(\frac{1}{2}\right)^{i-1} 2 d_{1}, & \text { for } i \leq n, \\ \left(\frac{1}{2}\right)^{i-n-1} 2 d_{n+1}, & \text { for } n+1 \leq i \leq m .\end{cases}
$$

The exact closed-form BER expression of generalized PAM and QAM is derived in [20]. Based on the constellation diagram of each modulation scheme, the decision region can be obtained for each bit. Thus by taking the integral over the decision region for each bit, the probability that the bit is decoded successfully as well as the error probability can be obtained. As the exact BER expression is very complicated, it is not easy to be used to formulate and solve our problem, and also the computational complexity of the resultant algorithm will be too high for an online scheduler. So, a BER approximation is needed. Since the exact BER expression is a summation of a series of complementary error function $\operatorname{erfc}()$, 


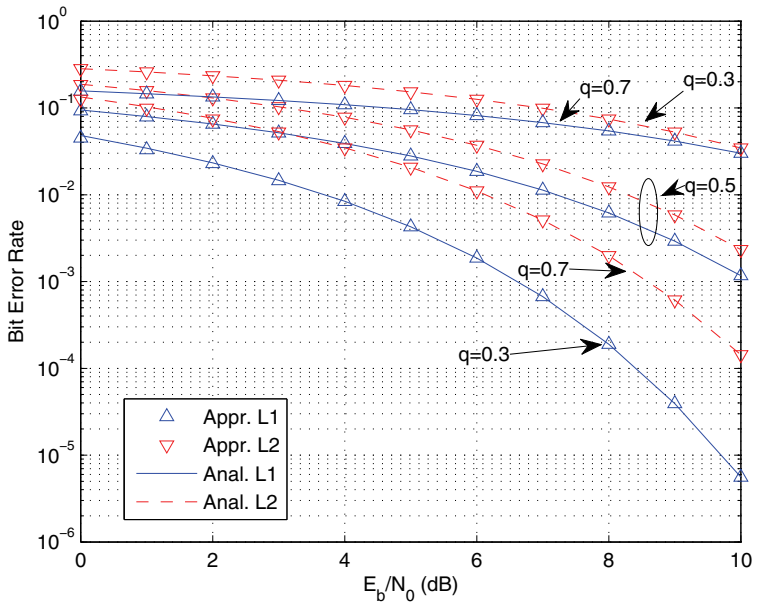

Fig. 2. Validation of BER approximation for 2/4-HMPAM with Gray mapping. $q$ is the energy portion of layer- 1 signal. By different $q$, we have different constellation diagram setting, which has different Euclidean distance among constellation points. The choice of $q$ is limited since $\forall i<j, d_{i}>d_{j}$.

which decades very fast, thus the BER is mainly determined by the shortest Euclidean distance between the corresponding constellation points to the decision boundaries.

For instance, the BER of the first and second bit in a 2/4HMPAM are, respectively

$$
P_{b, 2}^{(1)}=\frac{1}{4}\left[\operatorname{erfc}\left(\frac{d_{1}+d_{2}}{\sqrt{N_{0}}}\right)+\operatorname{erfc}\left(\frac{d_{1}-d_{2}}{\sqrt{N_{0}}}\right)\right],
$$

$P_{b, 2}^{(2)}=\frac{1}{2} \operatorname{erfc}\left(\frac{d_{2}}{\sqrt{N_{0}}}\right)+\frac{1}{4}\left[\operatorname{erfc}\left(\frac{2 d_{1}-d_{2}}{\sqrt{N_{0}}}\right)-\operatorname{erfc}\left(\frac{2 d_{1}+d_{2}}{\sqrt{N_{0}}}\right)\right]$

The BER of the first bit is mainly determined by the Euclidean distance between symbol 01 and 11, and similarly, the BER of the second bit is by that between symbol 00 and 01 (or symbol 11 and 10). Hence, their BER can be approximated by, respectively,

$$
\tilde{P}_{b, 4}^{(1)}=\frac{1}{4} \operatorname{erfc}\left(\frac{d_{1}-d_{2}}{\sqrt{N_{0}}}\right), \tilde{P}_{b, 4}^{(2)}=\frac{1}{2} \operatorname{erfc}\left(\frac{d_{2}}{\sqrt{N_{0}}}\right) .
$$

Fig. 2 shows the BER approximation for 2/4-PAM. From the figure, in all three configurations of 2/4-PAM, the approximations are close to the analytical results.

Such approximation only considers the dominant term in the exact BER expression, which can be named dominant term approximation. Given that the typical BER requirement is below $10^{-3}$, it is reasonable to use the dominant term approximation, whose accuracy has been confirmed in [21] for QAM in the low BER region.

Using the dominant term approximation and the constellation point distance (9), the BER of each bit in $2^{2 n} / 2^{2 m}$ HMsQAM can be calculated by

$$
\tilde{P}_{b, m}^{(i)}= \begin{cases}\frac{1}{2^{m+1-i}} \operatorname{erfc}\left(\frac{d_{i}-\sum_{j=i+1}^{m} d_{j}}{\sqrt{N_{0}}}\right), & \text { for } i=1, \ldots, m-1, \\ \frac{1}{2} \operatorname{erfc}\left(\frac{d_{i}}{\sqrt{N_{0}}}\right), & \text { for } i=m,\end{cases}
$$

where $\tilde{P}_{b, m}^{(i)}$ is the BER of the $i$-th bit in in-phase or quadrature and $m$ is the total number of bits transmitted in in-phase
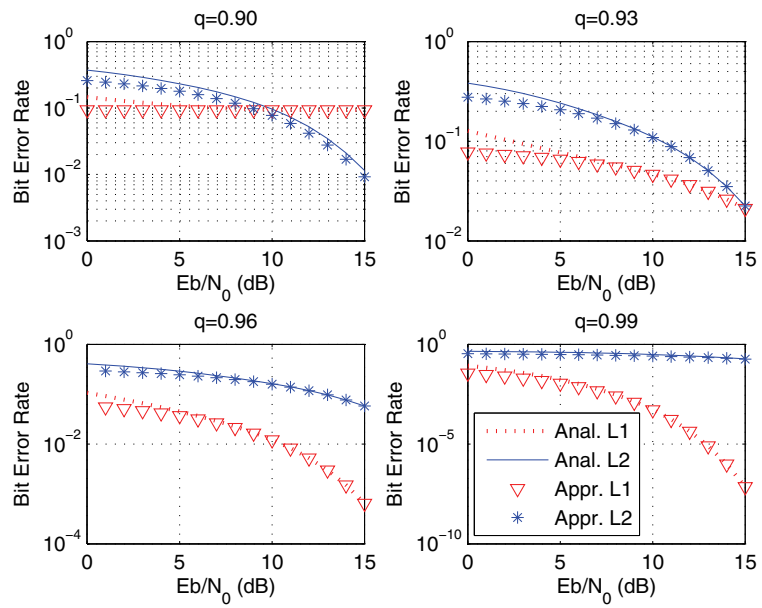

Fig. 3. Validation of BER approximation for 4/16-HMPAM with Gray mapping.

or quadrature. As layer-1 bits and layer-2 bits are transmitted to different users, whose received signal powers can be different, i.e., the Euclidean distances of the received signals of different users are different, thus we need to differentiate the corresponding Euclidean distances. In the following, $d_{i}$ and $d_{i,(j)}(j \in\{1,2\})$ are used to represent the Euclidean distance in the transmitted signal constellation diagram and that in the received signal constellation diagram of layer- $j$ user, respectively.

The BER of the layer-1 bits is

$$
\tilde{P}_{e}^{(1)}=\frac{1}{n} \sum_{i=1}^{n} \tilde{P}_{b, m}^{(i)}
$$

Substituting (10) into (11) with $d_{i,(1)}$ replacing $d_{i}$, after some manipulations, we obtain

$\tilde{P}_{e}^{(1)}=\frac{1}{n}\left[\left(\frac{1}{2}\right)^{m-n}-\left(\frac{1}{2}\right)^{m}\right] \operatorname{erfc}\left(\frac{d_{n,(1)}-\left(2^{m-n}-1\right) d_{m,(1)}}{\sqrt{N_{0}}}\right)$.

Similarly, the BER of the layer- 2 bits is

$$
\tilde{P}_{e}^{(2)}=\frac{1}{m-n}\left[1-\left(\frac{1}{2}\right)^{m-n}\right] \operatorname{erfc}\left(\frac{d_{m,(2)}}{\sqrt{N_{0}}}\right) .
$$

A sample validation of BER based on the dominant term approximation for 4/16-PAM is shown in Fig. 3. The dominant term approximation is accurate as long as the BER is lower than a threshold, e.g., $10^{-3}$.

The average symbol energy of the transmitted signal is $E_{s}^{(t)}=2 \sum_{i=1}^{m} d_{i}^{2}$, which consists two parts, the average symbol energy of layer- 1 signal and that of layer- 2 signal,

$$
\begin{gathered}
E_{s, 1}^{(t)}=2 \sum_{i=1}^{n} d_{i}^{2}, \\
E_{s, 2}^{(t)}=2 \sum_{i=n+1}^{m} d_{i}^{2} .
\end{gathered}
$$


For the average symbol energy of the transmitted signal and the received signal, we have

$$
\frac{E_{s, j}^{(t)} B N_{0}}{P}=\frac{E_{s, j}^{(r)}}{\gamma^{(j)}}, \quad j=1,2,
$$

where $\gamma^{(j)}$ is the channel SNR of layer- $j$ user.

Since the transmitted signal should satisfy the energy constraint, we have $E_{s}^{(t)} \leq P / B$. By substituting (9), (14), (15) and (16) into the energy constraint, we obtain

$$
\frac{4^{m-n}-1}{3}\left(\frac{d_{m,(2)}}{\sqrt{N_{0}}}\right)^{2} \frac{2}{\gamma^{(2)}}+\frac{4^{n}-1}{3}\left(\frac{d_{n,(1)}}{\sqrt{N_{0}}}\right)^{2} \frac{2}{\gamma^{(1)}} \leq 1 \text {. }
$$
is

Substituting (9) and (16) into (11), the BER of layer-1 bits

$$
\tilde{P}_{e}^{(1)}=\frac{2^{n}-1}{n 2^{m}} \operatorname{erfc}\left(\frac{d_{n,(1)}}{\sqrt{N_{0}}}-\left(2^{m-n}-1\right) \sqrt{\frac{\gamma^{(1)}}{\gamma^{(2)}}} \frac{d_{m,(2)}}{\sqrt{N_{0}}}\right) .
$$

Thus, the PF-utility maximization problem for a system with HMsQAM modulation can be formulated as follows.

Problem 2:

$$
\begin{array}{ll}
\max & \left(1+\frac{2 n}{(T-1) R^{(1)}}\right)\left(1+\frac{2(m-n)}{(T-1) R^{(2)}}\right) \\
\text { s.t. } & \tilde{P}_{e}^{(1)}<P_{e 1}, \tilde{P}_{e}^{(2)}<P_{e 2} \quad \text { and } \quad(17), \\
& m \geq n, \quad m, n \in[0,1, \ldots, K]
\end{array}
$$

where $R^{(j)}$ is the average rate of layer- $j$ user, $P_{e 1}$ and $P_{e 2}$ are the BER requirements of user 1 and 2, respectively, and $2^{2 K}$-HMsQAM is the maximal modulation scheme.

Using the dominant term approximation, the BER of the higher layer (generally for users with higher channel SNR) does not rely on the constellation distance in the lower layer, which dramatically simplifies the problem, not only for BER calculation, but also for modulation configuration.

\section{Scheduling Algorithm Design}

For the optimal scheduling algorithm design, the convexity of Problem 1 may not be equivalent to the convexity of PFutility function (5) and is generally unknown. Since Problem 1 is a one-dimensional problem in a closed set, the optimum solution can be obtained straightforwardly by comparing the points with zero derivative and the boundary points. In the following, we mainly focus on Problem 2, which is a more practical and difficult problem.

\section{A. Optimal Solution: $O^{2 U}$ HM PFS Algorithm}

Problem 2 is a mixed-integer programming problem which is generally hard to solve. However as the number of the available modulation schemes is limited in practice, it is feasible to solve such a problem by the searching algorithm shown in Algorithm 1. Based on possible bit allocation to different layer and the BER requirement of layer-2, we can obtain the Euclidean distance requirement of layer- 2 signal (lines 2 - 8). Based on the energy constraint and the BER constraint of layer-1 signal (lines $9-11$ ), we can decide whether it is a feasible solution. If it is feasible, then the utility is calculated and updated (lines 12 - 14). By searching all

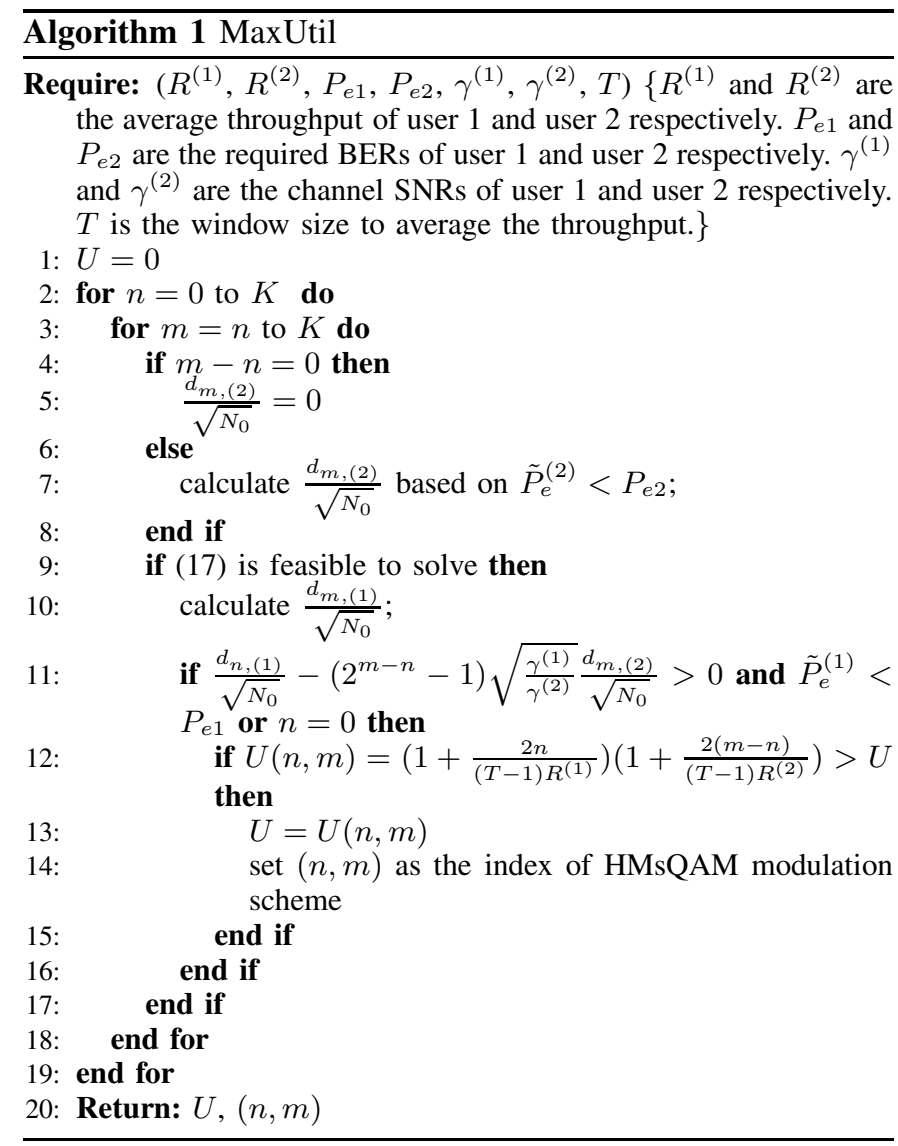

the possible bit allocation, we can find one with the maximal utility, which is the desired one.

Using Algorithm 1 to maximize the PF-utility of any given user pair, the scheduling problem can be solved by Algorithm 2, which is a simple exhaustive search over all user pairs. Note that in Algorithm 2, we do not explicitly calculate the maximal PF-utility when a single user is chosen to transmit. This is because the single user PF-utility can be calculated with Algorithm 1 when $n=0$ or $m=n$.

Both Algorithm 1 and Algorithm 2 can be further improved in reducing the computation time. For instance, if a BER look-up table is used to deal with the single user case in Algorithm 1, the computation time can be slightly reduced, but the order of computational complexity remains the same. Thus, we only present the original algorithm here.

The computational complexity of Algorithm 1 is $\mathcal{O}\left(K^{2}\right)$, where $K$ is the number of available modulation schemes, and it is generally a small number (typically less than 10 in a practical system). The computational complexity of Algorithm 2 is $\mathcal{O}\left(N^{2} K^{2}\right)$, where $N$ is the number of active users and can be a large number, so lower complexity algorithms with comparable performance to Algorithm 2 are desirable for the online scheduler.

\section{B. Suboptimal Solution: $S^{2 U}$ HM PFS Algorithm}

In order to reduce the computational complexity of the proposed optimal algorithm, we reconsider the PF-utility maximization Problem 1. Note that if $T$ is sufficiently large, the problem is approximated by a maximal weighted sum rate problem as follows. 


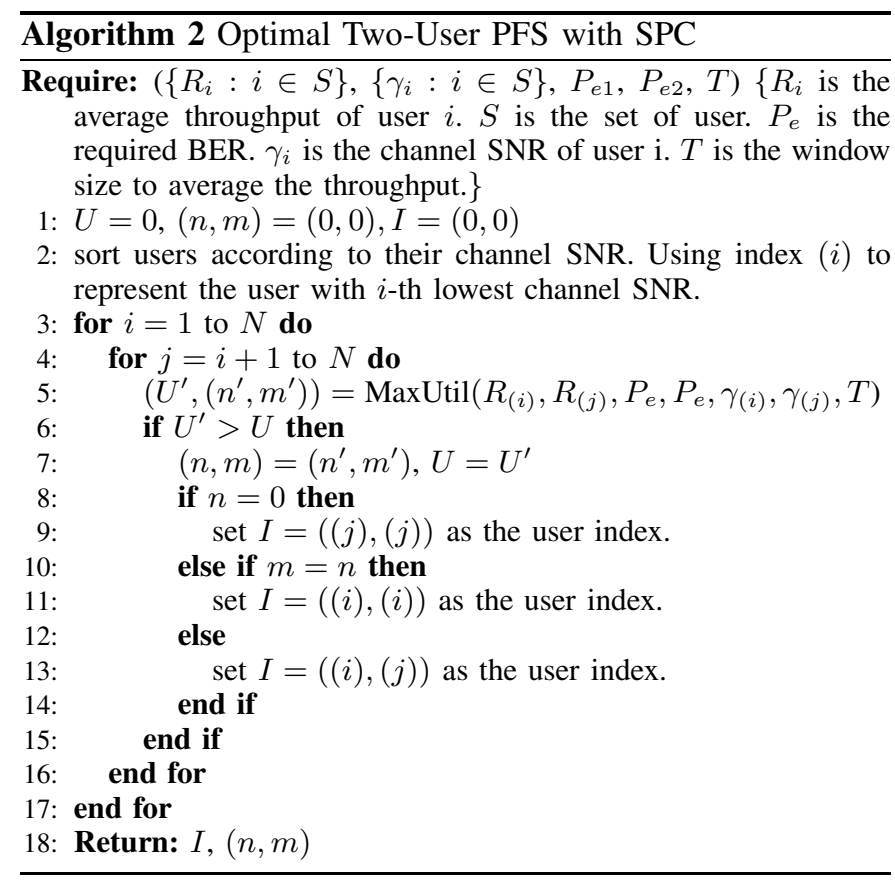

Problem 3:

$$
\begin{array}{ll}
\max & \frac{\log \left(1+q_{(i, j)}^{i} \gamma_{i}\right)}{R_{i}}-\frac{\log \left(1+q_{(i, j)}^{i} \gamma_{j}\right)}{R_{j}} \\
\text { s.t. } & 0 \leq q_{(i, j)}^{i} \leq 1
\end{array}
$$

By taking the derivative of the objective function and setting it to zero, we can obtain the optimal power allocation $q_{(i, j)}^{i *}$ and the corresponding maximal PF-utility $U_{(i, j)}^{*}$. When two users are selected, i.e., $q_{(i, j)}^{i *} \in(0,1)$, the maximal PF-utility is

$$
U_{(i, j)}^{*}=\frac{\log \left(1+\gamma_{j}\right)}{R_{j}}+G_{i, j}^{\mathrm{SPC}}
$$

where

$$
G_{i, j}^{\mathrm{SPC}}=\frac{1}{R_{i}} \log \left(\frac{R_{j}}{\gamma_{j}} \frac{\gamma_{i}-\gamma_{j}}{R_{i}-R_{j}}\right)-\frac{1}{R_{j}} \log \left(\frac{R_{i}}{\gamma_{i}} \frac{\gamma_{i}-\gamma_{j}}{R_{i}-R_{j}}\right)
$$

is the SPC gain.

Recall that the single user PFS is to select the user with the largest $\log (1+\gamma) / R$, which is identical to the first term of (19). Thus, we can separate the user selection and resource allocation by using single-user PFS to find the first user, which is the low SNR user (lines 2 - 4) and by using multi-user PFS to find the second user which is the high SNR user (lines 8 - 20). Note that such decomposition cannot guarantee the optimality, since the largest $\log (1+\gamma) / R$ may not necessarily lead to the maximal PF-utility among all possible user pairs, so it is a suboptimal solution. Following a similar approach, a heuristic suboptimal algorithm can be developed to solve Problem 2, as shown in Algorithm 3. Note that, if the user selected by single-user PFS has the maximal channel SNR, only one user will be selected and the bit allocation can be done by a single-user BER look-up table (lines 5 - 7). The computational complexity of Algorithm 3 is $\mathcal{O}\left(N K^{2}\right)$ only, which is one order lower than that of Algorithm 2.

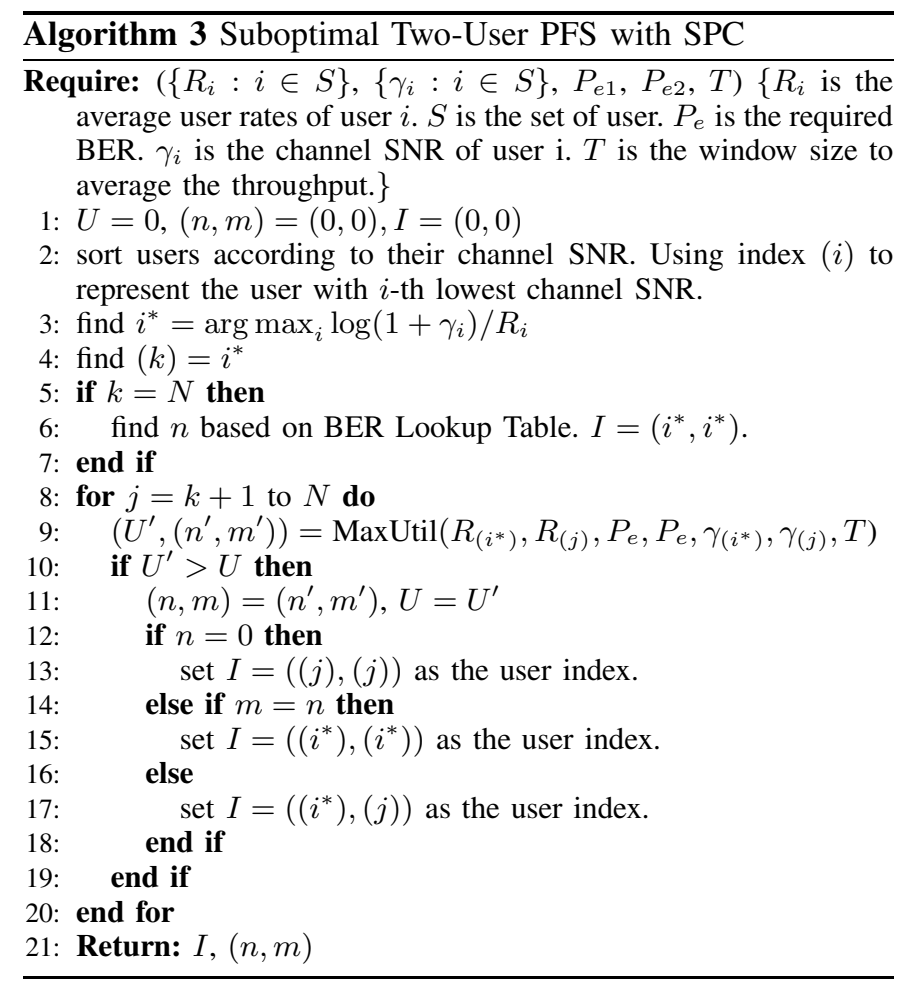

\section{Further Discussion: J-Layer HM problem}

We can further consider a more general scheduling problem with $J$-layer HM, which becomes much more complicated as discussed below. To find the optimal solution, we need to search all the possible resource allocation strategies and test whether it is feasible under the energy and BER constraints. By observing the procedure of BER approximation, we can conclude that, the expression of the approximated BER of a higher layer is not related to the lower layers. Thus, for any bit allocation scheme (allocating the number of bits to each layer (user)), we can tell whether the bit allocation is feasible by iteratively solving the BER constraints and test the energy constraint. The computational complexity for a given group of users is at least $\Omega\left(\log (K)^{J}\right)$ for the $J$-Layer HM. To find the optimal utility, we need to search all $\frac{N !}{J !(N-J) !}$ user groups, thus the overall computational complexity is at least $\Omega\left(\log (K)^{J} \frac{N !}{J !(N-J) !}\right)$. Note that, without the BER approximation, the problem is even much harder since it is not easy to test whether the bit allocation is feasible due to the coupled BER expressions for different layers.

We can also generalize the sub-optimal Algorithm 3 for $J$-layer HM, by selecting the layer-1 user based on the single-user PFS first, then selecting the higher layer's users sequentially based on the previously selected users and the $i$ user PFS criteria. On average, the number of candidate users for the first layer user is $N$, and the number of candidate users for the second layer user is $N / 2$ (since only the users having higher SNRs than the first user will be searched), and the number of candidate users for the $J$-th layer user is $N / 2^{J-1}$. Thus, on average the number of candidate user groups is $\frac{N^{J}}{2^{(J-1) J / 2}}$. The average computational complexity is then at least $\Omega\left(\log (K)^{J} \frac{N^{J}}{2^{(J-1) J / 2}}\right)$, which is much smaller compared to that of the optimal solution. Obviously, this 
TABLE I

PARAMETER SETTING.

\begin{tabular}{|c|c|c|c|c|c|}
\hline Parameters & $C$ & $\beta$ & $\sigma_{\phi_{d B}}$ & $l_{0}$ & $h_{t}$ \\
\hline Values & $-31.45 \mathrm{~dB}$ & 3.71 & $3.5 \mathrm{~dB}$ & $1 \mathrm{~m}$ & $10 \mathrm{~m}$ \\
\hline Parameters & $h_{r}$ & $P_{t}$ & $N_{0}$ & $B$ & $r$ \\
\hline Values & $1 \mathrm{~m}$ & $10 \mathrm{dBm}$ & $-174 \mathrm{dBm} / \mathrm{Hz}$ & $500 \mathrm{KHz}$ & $300 \mathrm{~m}$ \\
\hline
\end{tabular}
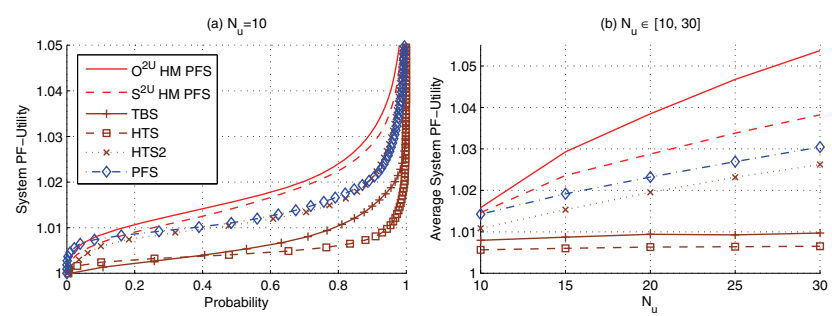

Fig. 4. System PF-utility comparison.

heuristic solution is suboptimal and its performance requires further investigation.

\section{Performance Evaluation}

Extensive simulations have been conducted to evaluate the performance of the proposed algorithms. For comparison, the performance of four existing state-of-the-art scheduling algorithms, including the single-user PFS [4], HTS [15], TBS [14] and HTS2 [17] is also evaluated by simulations.

\section{A. Simulation Settings}

We consider the downlink of a single-cell narrowband cellular network and assume the wireless channel is a quasi-static Rayleigh fading channel. A classic path loss and shadowing model is used to model the large-scale propagation effects [12]: $\frac{P_{r}}{P_{t}}(d B)=10 \log _{10} C-10 \beta \log _{10} \frac{l}{l_{0}}-\phi_{d B}$, where $P_{r}$ is the received power, $P_{t}$ is the transmitted power, $C$ is a unit-less constant which depends on the characteristics of the antenna and the average channel attenuation, $\beta$ is the path loss exponent, $l$ is the distance between the transmitter and the receiver, $l_{0}$ is the reference distance, and $\phi_{d B}$ is a Gaussian distributed R.V. with zero mean and variance of $\sigma_{\phi_{d B}}^{2}$.

Table I summarizes the parameters of an urban macrocell in [12] and other parameters used in simulation, including antenna height of the transmitter $\left(h_{t}\right)$ and the receiver $\left(h_{r}\right)$, maximal transmission power $\left(P_{t}\right)$, noise spectral density $\left(N_{0}\right)$, channel bandwidth $(B)$ and radius of cell $(r)$. The single-user modulation schemes are $2^{2 n}$-QAM and $2^{n}$-PAM, $\forall n \in\{1,2,3,4\}$, and the multi-user modulation schemes are $2^{2 n} / 2^{2 m}$-HMsQAM, $\forall n, m \in\{1,2,3,4\}, m>n$. The BER requirements are all set to be $10^{-3}$. The simulation evaluates 400 random user deployments and each deployment has 2000 time slots. The throughput for each deployment is the average over 2000 time slots. The window size $T$ for the PFS algorithms is 1000 as suggested in [23]. We vary the number of users $\left(N_{u}\right)$ to simulate a wide range of scenarios.

\section{B. System PF-Utility Comparison}

In Sec. IV, we propose an optimal algorithm and a suboptimal algorithm to solve Problem 2. The optimality of the
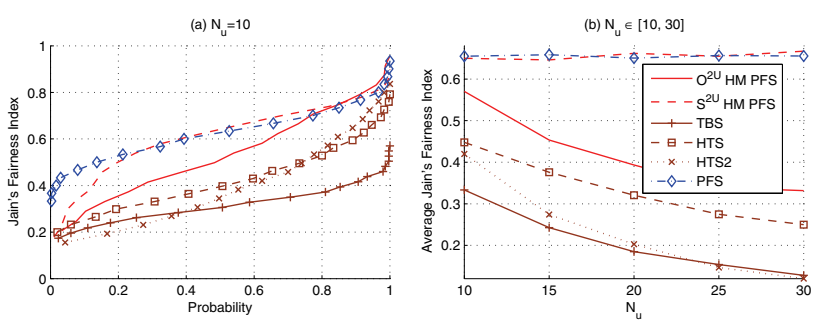

Fig. 5. Jain's fairness index comparison.

algorithms can be judged by the system PF-utility. From the definition, the maximal system PF-utility leads to the best proportional fairness, which measures the tradeoff between efficiency and fairness. The system PF-utilities are compared in Fig. 4, where the y-axis is the system PF-utility and the $\mathrm{x}$-axis represents the probability that the PF-utility is below certain value. A higher curve indicates a higher PF-utility. As shown in Fig. 4 (a), the system PF-utility of the optimal $\mathrm{O}^{2 \mathrm{U}} \mathrm{HM}$ PFS is strictly higher than that of the suboptimal $\mathrm{S}^{2 \mathrm{U}}$ HM PFS. Both of the proposed algorithms outperform the single-user PFS substantially, as they can use HM to explore the extra capacity, which moves the system operating point towards a higher PF-utility. On the other hand, the system PF-utilities of the proposed scheduling algorithms are much higher than that of HTS and TBS, the two greedy scheduling algorithms for HM transmissions. Thus, without PFS, even though HTS and TBS can use HM to achieve a higher throughput for some users, the overall system PF-utility is not improved. The proposed algorithms also outperform HTS2 which is the best multi-user scheduling algorithm in the literature. Although HTS2 can utilize the extra capacity region thanks to HM, it is unable to achieve the best PF-utility, due to the choice of the second user. The average system PF-utilities w.r.t. the number of users are compared in Fig. 4 (b). It can be seen that, the PF-utilities of PFS-based algorithms are higher with the increment of $N_{u}$. For the two greedy algorithms, TBS and HTS, $N_{u}$ has almost no positive impact on the PF-utility. Next, we compare their performance in terms of fairness and system throughput separately.

\section{Fairness Comparison}

1) Jain's Fairness Index: Jain's fairness index has been widely used to measure the fairness, which is represented as $\mathcal{J}\left(x_{1}, x_{2}, \ldots, x_{N_{u}}\right)=\frac{\left(\sum_{i=1}^{N_{u}} x_{i}\right)^{2}}{N_{u} \cdot \sum_{i=1}^{N_{u}} x_{i}^{2}}$, where $x_{i}$ is user $i$ 's throughput.

In Fig. 5 (a), where the y-axis is the Jain's fairness index in terms of per-user throughput and the x-axis is its Cumulative Distribution Function (CDF). Each point in the curve illustrates the probability that the fairness index is below certain threshold. The scheduler corresponding to a higher curve achieves better fairness. Overall, the proposed HM PFS and PFS schemes have the best fairness performance among all multi-user scheduling algorithms. For the TBS, HTS, and HTS2 algorithms, they all tend to favor the users with better channel quality, which results in worse fairness.

The average Jain's fairness index w.r.t. the number of users is compared in Fig. 5 (b). The Jain's index of all schemes 


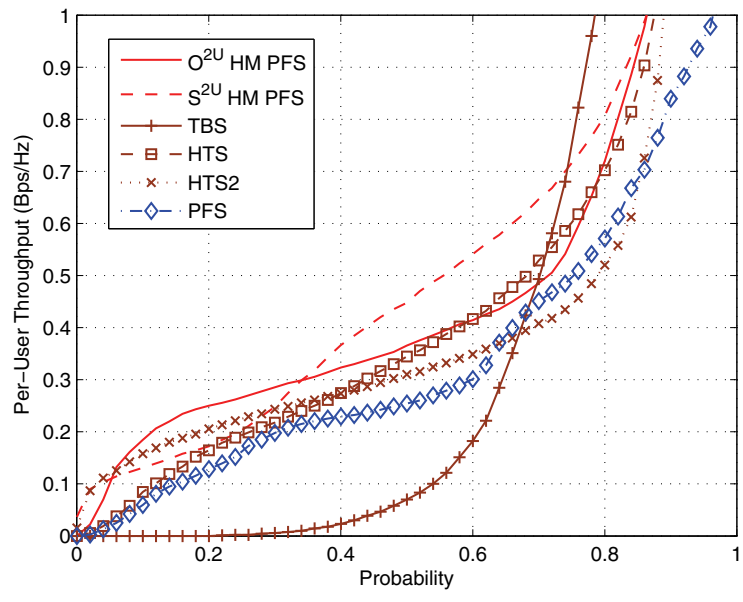

Fig. 6. Per-user throughput distribution, $N_{u}=10$.

except PFS and $\mathrm{O}^{2 \mathrm{U}} \mathrm{HM}$ PFS will decrease with a larger number of users, which means more unfairness for the users with a low SNR. The PFS and $\mathrm{O}^{2 \mathrm{U}} \mathrm{HM}$ PFS can maintain a good Jain's fairness independent of the number of users.

The limitation of Jain's fairness index is that it does not consider the heterogeneity of users, and it is not desirable for the system where the user is not charged solely based on the throughput. In a practical system, the distribution of per-user throughput is a more meaningful metric for both fairness and user-perceived QoS. Next, we consider the CDF function of per-user throughput.

2) CDF Function of Per-User Throughput: If the performance of the worst-user is improved, there will be more satisfied users with higher overall user-perceived QoS. Given the performance of the $\alpha \%$ worst users (who have the throughput lower than $1-\alpha \%$ of the total users), we can judge whether the scheduler is worst-user friendly or not.

In Fig. 6, the y-axis is the per-user throughput, and the $\mathrm{x}$-axis is its CDF. As shown in the figure, with the TBS algorithm, about $30 \%$ users can be starving (with zero throughput), as TBS focuses on maximizing the total throughput only. Compared to TBS, HTS uses a different second-user selection criterion which significantly reduces the percentage of starving users, so its performance is close to PFS. HTS2, which is a modified algorithm of HTS, can achieve an even better performance than that of PFS when $\alpha$ is small. However, the per-user throughput of about $80 \%$ of users under HTS2 is below $0.5 \mathrm{bps} / \mathrm{Hz}$, which is worse than PFS.

Overall, in terms of $\alpha \%$ worst-user friendliness, the proposed $\mathrm{O}^{2 \mathrm{U}} \mathrm{HM}$ PFS and $\mathrm{S}^{2 \mathrm{U}} \mathrm{HM}$ PFS algorithms are the best two algorithms, for majority of cases when $\alpha \leq 60$. It means that the users with worse channel conditions can achieve higher throughput using the proposed two algorithms than the previous solutions. The question is whether they achieve the worst-user friendliness at the cost of the system efficiency, which will be investigated in the following subsection.

\section{System Throughput}

Here, system throughput is used to measure the efficiency. The comparison is based on two network configurations with different number of users $N_{u}$, and the results are shown

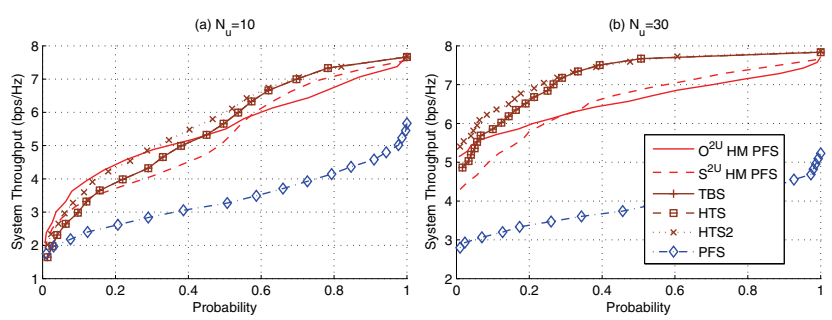

Fig. 7. System throughput.

in Fig. 7. The y-axis represents the system throughput, and the $\mathrm{x}$-axis is its $\mathrm{CDF}$, and a higher curve corresponding to better throughput performance. In Fig. 7 (a), the two best-user selection based greedy scheduling algorithms, HTS and TBS, achieve almost identical throughput so the two curves overlap with each other. Since both of the schemes cannot fully explore the benefit of SPC/HM, the throughput is not always the best. The HTS2 achieves the highest throughput for about $60 \%$ of the cases. The throughputs of TBS, HTS and HTS2 converge in the tail part. $\mathrm{O}^{2 \mathrm{U}}$ HM PFS can achieve the highest system throughput for $30 \%$ cases. The average system throughput gap between $\mathrm{S}^{2 \mathrm{U}} \mathrm{HM}$ PFS and $\mathrm{O}^{2 \mathrm{U}} \mathrm{HM}$ PFS is less than $5 \%$.

Comparing the results in Figs. 7 (a) and (b), with the increment of the number of users, $N_{u}$, using the four multiuser scheduling algorithms can substantially increase the system throughput (more than $50 \%$ on average). The throughput increment of TBS, HTS and HTS2 are larger than the two proposed PFS algorithms. This is because a greedy algorithm tends to select the user with the best channel quality, which leads to a higher throughput. Comparing Figs. 7 (a) and 6, the higher system throughput of HTS2 is contributed mainly by $15 \%$ of the best users, which concludes the fairness concern for HTS2.

In summary, by using multi-user scheduling with HM, the system throughput can be improved substantially. For instance, as shown in Fig. 7 (b), on average, the system throughput with $\mathrm{HM}$ is increased by $80 \%$ compared with the case without HM (i.e., single-user PFS). Furthermore, the proposed $\mathrm{O}^{2 \mathrm{U}} \mathrm{HM}$ PFS and $\mathrm{S}^{2 \mathrm{U}} \mathrm{HM}$ PFS can achieve a comparable system throughput as the other three multiuser scheduling algorithms, TBS, HTS and HTS2. Different from TBS, HTS and HTS2, which suffer from starvation and unfairness problems, the proposed scheduling algorithms can maintain good fairness, so users can experience more consistent and satisfactory QoS.

\section{E. Access Delay}

Beside the throughput and fairness, delay is also an important QoS metric. The link-layer delay includes queueing delay, transmission delay and access delay. The queueing delay and transmission delay depend on the per-user throughput, i.e., a higher throughput means a higher service rate, which results in a smaller queueing plus transmission delay. Since the proposed algorithms are worst-user friendly in terms of peruser throughput, they should be worst-user friendly in terms of queueing and transmission delay too. Thus, here we focus on the access delay.

Access delay is defined as the number of time slots that a head-of-queue packet has to wait till it is transmitted. A 


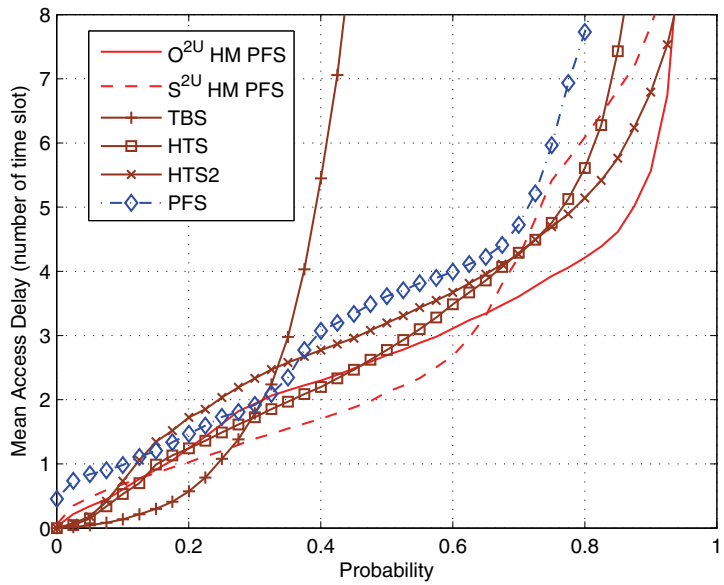

Fig. 8. Access delay, $N_{u}=10$.

large access delay may have adverse impacts on upper-layer protocol performance, such as leading to a time-out event of a TCP connection.

The average access delay performance is compared in Fig. 8. The $y$-axis represents the average access delay of a user, and the $\mathrm{x}$-axis is its CDF. Overall, the access delay using a multi-user scheduling (except TBS) can be reduced as two users are scheduled to transmit per-slot. TBS suffers severe unfairness and starvation problem: around $30 \%$ of the users have a very small access delay while the majority of the users have the access delay even longer than that with single-user PFS.

\section{CONCLUSION AND FurTher Discussion}

In this paper, we have investigated the opportunistic PFS scheduling in an HM-aided wireless network. A Shannon capacity-based theoretical utility maximization problem considering SPC and an HM-based utility maximization problem have been formulated. The former provides insights on theoretical system performance bounds and guidelines for solving the latter which is the focus of this work. An optimal scheduling algorithm $\mathrm{O}^{2 \mathrm{U}}$ HM PFS and a low-complexity suboptimal scheduling algorithm $\mathrm{S}^{2 \mathrm{U}}$ HM PFS have been proposed. We have evaluated the performance of the proposed algorithms, compared with other state-of-the-art HM based multi-user scheduling algorithms and the single-user opportunistic PFS algorithm. Simulation results have shown that both $\mathrm{O}^{2 \mathrm{U}}$ HM PFS and $\mathrm{S}^{2 \mathrm{U}}$ HM PFS can increase the system throughput substantially, while the fairness which is critical to user-perceived QoS can be well maintained.

To optimize the design of scheduler for wireless networks, there are many open issues beckoning for further research. First, although CSI feedback in PFS has been well investigated in [22], the discussion should be extended to the multi-user PFS case. Second, the performance of the heuristic algorithm for $J$-layer HM case should be further investigated. Third, how to extend the solution to a coded HM-based system and to consider HARQ requires further investigation. A possible approach is by discretizing the power allocation to constrain the number of transmission modes (i.e., modulation and coding schemes given the power allocation) then obtaining a SNRtransmission mode mapping. However, this approach cannot be applied to the case with coded modulation (such as trelliscoded modulation) directly. Finally, as another important feature of the wireless channel is the frequency selective fading, how to jointly explore frequency diversity, multi-user diversity, SPC gain and maintain fairness to optimize the wireless scheduling algorithm design will be the ultimate goal in this area.

\section{REFERENCES}

[1] R. Knopp and P. Humblet, "Information capacity and power control in single-cell multiuser communications," in Proc. 1995 IEEE ICC.

[2] D. Tse, "Optimal power allocation over parallel Gaussian broadcast channels," in Proc. 1997 ISIT.

[3] F. Kelly, A. Maulloo, and D. Tan, "Rate control for communication networks: shadow prices, proportional fairness and stability," J. Operational Research Society, vol. 49, no. 3, pp. 237-252, Mar. 1998.

[4] P. Viswanath, D. Tse, and R. Laroia, "Opportunistic beamforming using dumb antennas," IEEE Trans. Inf. Theory, vol. 48, no. 6, pp. 1277-94, June 2002.

[5] T. Cover, "Broadcast channels," IEEE Trans. Inf. Theory, vol. 18, no. 1, pp. 2-14, Jan. 1972.

[6] M. Sharif, A. F. Dana, and B. Hassibi, "Differentiated rate scheduling for Gaussian broadcast channels," in Proc. 2005 ISIT.

[7] X. Wang, W. Chen, and Z. Cao, "SPARC: superposition-aided rateless coding in wireless relay systems," IEEE Trans. Veh. Technol., vol. 60, no. 9 , pp. 4427-38, Nov. 2011

[8] L. Cai, S. Xiang, Y. Luo, and J. Pan, "Scalable modulation for video transmission in wireless networks," IEEE Trans. Veh. Technol., vol. 60, no. 9, no. 4314-23, Nov. 2011.

[9] Z. Yang, L. Cai, Y. Luo, and J. Pan, "Topology-aware modulation and error-correction coding for cooperative networks," IEEE J. Sel. Areas Commun., vol. 30, no. 2, pp. 379-387, Feb. 2012.

[10] Z. Yang, Y. Luo, and L. Cai, "Network modulation: a new dimension to enhance wireless network performance," in Proc. 2011 IEEE Infocom.

[11] H. Kim and Y. Han, "A proportional fair scheduling for multicarrier transmission systems," IEEE Commun. Lett., vol. 9, no. 3, pp. 210-212, Mar. 2005.

[12] A. Goldsmith, Wireless Communications. Cambridge University Press, 2005.

[13] Digital Video Broadcasting (DVB) Framing Structure, Channel Coding and Modulation for Digital Terrestrial Television (DVB-T) V1.5.1, ETSI Standard ETS 300 744, Nov. 2004.

[14] M. Hossain, M.-S. Alouini, and V. Bhargava, "Rate adaptive hierarchical modulation-assisted two-user opportunistic scheduling," IEEE Trans. Wireless Commun., vol. 6, no. 6, pp. 2076-2085, June 2007.

[15] M. Hossain, M.-S. Alouini, and V. Bhargava, "Two-user opportunistic scheduling using hierarchical modulations in wireless networks with heterogenous average link gains," IEEE Trans. Commun., vol. 58, no. 3 , pp. 880-889, Mar. 2010.

[16] A. Jdidi and T. Chahed, "Impact of hierarchical modulation on proportional fair in OFDMA-based networks," in Proc. 2010 ComNet.

[17] A. Agustin, J. Vidal, and O. Muoz, "Performance of downlink schedulers with superposed or orthogonal transmissions," in Proc. 2010 IEEE ICC.

[18] R. Tyrrell Rockafellar, Convex Analysis. Princeton University Press, 1970.

[19] N. Jindal, S. Vishwanath, and A. Goldsmith, "On the duality of Gaussian multiple-access and broadcast channels," IEEE Trans. Inf. Theory, vol. 50, no. 5, pp. 768-783, May 2004.

[20] P. K. Vitthaladevuni and M.-S. Alouini, "A closed-form expression for the exact BER of generalized PAM and QAM constellations," IEEE Trans. Commun., vol. 52, no. 5, pp. 698-700, May 2004.

[21] L. Yang and L. Hanzo, "A recursive algorithm for the error probability evaluation of M-QAM," IEEE Commun. Lett., vol. 4, no. 10, pp. 304306, Oct. 2000.

[22] D. Gesbert and M.-S. Alouini, "How much feedback is multi-user diversity really worth?" in Proc. 2004 IEEE ICC.

[23] A. Jalali, R. Padovani, and R. Pankaj, "Data throughput of CDMAHDR a high efficiency-high data rate personal communication wireless system," in Proc. 2000 IEEE VTC - Spring.

[24] L. Xu, X. Shen, and J. W. Mark, "Dynamic fair scheduling with QoS constraints in multimedia W-CDMA cellular networks," IEEE Trans. Wireless Commun., vol. 3, no. 1, pp. 60-73, Jan. 2004. 
[25] C. Ng, K. Shum, C. Sung, and T. Lok, "A layered decomposition framework for resource allocation in multiuser communications," IEEE Trans. Veh. Technol., vol. 60, no. 2, pp. 729-733, Feb. 2011.

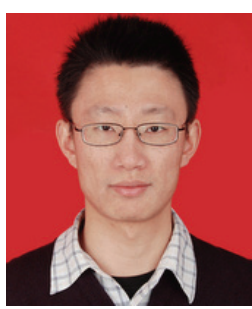

Xuan Wang (S'09) received the B.Eng. degree in information security and the M.S. degree in signal and information processing from Beijing University of Posts and Telecommunications, Beijing, China, in 2007 and 2010, respectively. He is currently working toward a Ph.D. degree with the Department of Electrical and Computer Engineering, University of Victoria, Canada. His current research interests include scheduling, resource allocation and crosslayer design in wireless networks.

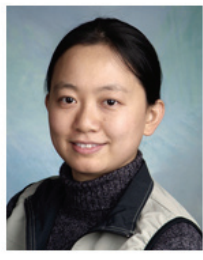

Lin Cai (S'00-M'06-SM'10) received her M.A.Sc. and $\mathrm{PhD}$ degrees (awarded Outstanding Achievement in Graduate Studies) in electrical and computer engineering from the University of Waterloo, Waterloo, Canada, in 2002 and 2005, respectively. Since 2005, she has been an Assistant Professor and then an Associate Professor with the Department of Electrical \& Computer Engineering at the University of Victoria. Her research interests span several areas in wireless communications and networking, with a focus on network protocol and architecture design supporting emerging multimedia traffic over wireless, mobile, ad hoc, and sensor networks.

She has been a recipient of the NSERC Discovery Accelerator Supplement Grant in 2010, the best paper award of IEEE ICC 2008, and the best academic paper award of IEEE WCNC 2011. She has served as a TPC symposium cochair for IEEE Globecom'10 and Globecom'13, and the Associate Editor for IEEE TRANSACTIONS ON WIRELESS COMMUNICATIONS, IEEE TRANSACTIONS ON VehicUlar TECHNOLOgY, EURASIP Journal on Wireless Communications and Networking, International Journal of Sensor Networks, and Journal of Communications and Networks (JCN). 